\title{
Does the old-fashioned sofosbuvir plus ribavirin treatment in genotype 2 chronic hepatitis $C$ patients still works for Koreans?
}

\author{
Jong Eun Yeon \\ Department of Internal Medicine, Korea University College of Medicine, Seoul, Korea
}

Keywords: Chronic hepatitis C; Genotype 2; Sofosbuvir; Ribavirin

\section{See Article on Page 311}

According to the 2017 Korean Association for the Study of the Liver (KASL) treatment guidelines of chronic hepatitis $C_{\text {, }}^{1}$ treatment options for genotype 2 patients are sofosbuvir plus ribavirin $(\mathrm{SOF}+\mathrm{R})$, SOF plus daclatasvir, glecaprevir plus pibrentasvir, SOF plus velpatasvir and pegylated interferon alpha plus ribavirin (PEG-IFN+R). Except in cases where only patients who cannot use the direct acting antivirals (DAAs), PEG-IFN+R is replaced by DAAs.

In reality, there are two possible DAA treatment options, SOF $+R$ (daily $400 \mathrm{mg}$ of SOF+1,000 or 1,200 mg/d of ribavirin if body weight less or over than $75 \mathrm{~kg}$ ) which can get insurance benefits and glecaprevir plus pibrentasvir which could not be used at the start of the study, those are currently available or are likely to be available for genotype 2 in Korea.

But in other continents, SOF+R treatment is no longer recommended because of frequent side effects of ribavirin and somewhat lower sustained virologic response (SVR) rate than other DAA combinations. ${ }^{2,3}$
Regarding SVR rate of SOF $+R$ treatment, previous studies conducted in Western countries have shown that SVR rates in the order of $80-95 \% .^{4-7}$ In a trial for the treatment-naïve genotype 2 patients comparing 12 weeks of SOF+R with 24 weeks of PEGIFN $+R$, ${ }^{4}$ SVR rate was $97 \%$ and $78 \%$ in each treatment group. In patient with and without liver cirrhosis, SVR rates were $91 \%$ and $98 \%$, respectively. For patients ineligible or intolerant to PEGIFN $+R$, SOF+R treatment achieved $93 \%$ of SVR. ${ }^{6}$ In IFN non-responders, 12 - or 16 -weeks of SOF+R treatment leads to $86 \%$ and $94 \%$ of SVR, respectively. In that study, SVR rates were $60 \%$ and $78 \%$ in patients with cirrhosis treated for 12 - and 16 -weeks of $\mathrm{SOF}+\mathrm{R}$ treatment, respectively. In another study including genotype 2 treated with 12 weeks of SOF+R, ${ }^{5}$ SVR rates were $97 \%$, $100 \%, 94 \%$, and $78 \%$ in treatment-naïve non-cirrhotics, treatment-naïve cirrhotics, treatment-experienced non-cirrhotics, and treatment-experienced cirrhotics, respectively.

These results suggested that treatment-experienced patients with cirrhosis may benefit from more than 12 weeks of therapy.

A real-world study showed that SVR rates of 12 or 16 -week of SOF+R for treatment-naïve genotype 2 cirrhotics were $72 \%$ and $100 \%$, respectively. ${ }^{8}$ In treatment-experienced cirrhotic patients,

\section{Abbreviations:}

DAAs, direct acting antivirals; KASL, Korean Association for the Study of the Liver; PEG-IFN, pegylated interferon alpha; $R$, ribavirin; SOF, sofosbuvir; SVR, sustained virologic response

\section{Corresponding author : Jong Eun Yeon}

Department of Internal Medicine, Korea University College of Medicine, 73 Inchon-ro, Seongbuk-gu, Seoul 02841, Korea

Tel: +82-2-2626-1030, Fax: +82-2-2626-1038

E-mail: jeyyeon@hotmail.com

https://orcid.org/0000-0002-0510-7371

Received : Sep. 2, 2018 / Accepted : Sep. 7, 2018 
SVR rates were $87 \%$ and $77 \%$ in 12 - or 16 -weeks treated group, respectively. In another real-world experience including 16- or 20-weeks of SOF+R for cirrhotics (including $51 \%$ of treatment experience), SVR rates were $95 \%$ and $91 \%$, respectively. ${ }^{9}$ An openlabel phase 3 study including treatment-experienced genotype 2 cirrhotic patients has shown that SVR rates were $87 \%$ and $100 \%$ for those receiving 16 - and 24 -weeks of $S O F+R$, respectively. ${ }^{10}$

Unlike the findings in the Western countries, studies conducted in Asia have shown that 12-week SOF+R leads to SVR rates of 96 $100 \%$, regardless of treatment experience or presence of cirrhosis. $^{11-13}$

In a phase 3b study conducted in Korea (treatment-experienced patients $18 \%$, liver cirrhosis $10 \%$ ), ${ }^{11}$ the overall SVR rate following 12 weeks of SOF+R was $97 \%$, and all of treatment-experienced patients including cirrhotics achieved SVR of $100 \%$. In another Korean real-life study, ${ }_{1}^{14}$ the SVR rates following 12 weeks of sofosbuvir and ribavirin were $98 \%$ (177/181) in treatment-naïve patients without liver cirrhosis and 97\% (32/33) among treatmentexperienced patients without liver cirrhosis. The SVR rate after 16 weeks of sofosbuvir and ribavirin therapy was $96 \%$ (50/52) in patients with liver cirrhosis, although it was not possible to distinguish between treatment-naive and treatment-experienced patients in that analysis.

In a phase 3 study conducted in Taiwan (treatment-experienced patients $50 \%$, liver cirrhosis $15 \%)_{1}^{12}$ the SVR rate following 12 weeks of SOF+R was $100 \%$, regardless of treatment experience or presence of cirrhosis. In a Japanese study, ${ }_{1}^{13}$ the SVR rate following 12 weeks of SOF+R was $97 \%$. The SVR rates of treatmentnaive and treatment-experienced patients were $98 \%$ and $95 \%$, respectively. Among them, all of eight treatment-naïve patients and 8 of 9 treatment-experienced cirrhotics achieved SVR. Overall, 16 of 17 patients (94\%) with cirrhosis achieved SVR.

Regarding SOF+R drug safety, the combination of SOF and ribavirin exhibited acceptable safety profiles. The rate of premature discontinuation because of adverse events was $1-2 \%$, that was not different from the placebo group. Adverse events associated with ribavirin including fatigue, insomnia, pruritus, and anemia were more frequent. The rate of moderate to severe adverse events (Grade 3 or 4) was 1-6\%. ${ }^{4-6}$

In this issue of Clinical and Molecular Hepatology, the retrospective study by Kim et al. including 163 genotype 2 patients (liver cirrhosis 30\%, treatment-experienced patients 12\%) treated with SOF+R for 12- or 16- weeks adjusted for presence of cirrhosis showed high SVR rate of $98.8 \%(161 / 163))^{15}$ Among 49 liver cirrhotic patients, 18 patients were treated for 12 weeks and all of them achieved SVR. Except one patient who stopped the treatment after 2 weeks of treatment because of severe anemia, only one treatment-naïve, non-cirrhotic patient showed relapse. Adverse events during the treatment were reported in $16 \%$ of patients. Eleven percent of patients needed to reduce ribavirin dosage because of anemia. Although this retrospective multicenter study has limitations, the authors concluded that 12- or -16 weeks of SOF+R was effective and well tolerated in both of treatmentnaïve and treatment-experienced Korean genotype 2 patients with or without cirrhosis.

As aforementioned, the studies conducted in Western countries have shown that overall SVR rate approximated $80-95 \%$ in Europe and North America. In contrast, SVR rates in Asia were closer to $100 \%$. It is unclear why the Westerns and Asians show different treatment response, but it can be explained as follows: When the baseline characteristics between studies from Western and Asian countries were compared, the average age and the rate of baseline high viral load were similar. The average body mass index (BMI) was 26-29 in the pivotal trials ${ }^{4-6}$ and 24 in Korea. ${ }^{11}$ In VALENCE study including SOF plus ribavirin for genotype $2,{ }^{5} \mathrm{pa}-$ tients with baseline BMI less or more than 30 had $97 \%$ and $75 \%$ of SVR, respectively. In that study, patients with- or without ribavirin dose reduction/interruption had SVR rates of $100 \%$ and $93 \%$, respectively. On the contrary, FISSION study ${ }^{4}$ including SOF plus ribavirin for genotype 2 had shown that ribavirin exposure was a significant factor for SVR (odds ratio 1.258). Kim et al.'s study did not include data of baseline BMI and prior ribavirin-exposure in their study populations. ${ }^{15}$ Men comprised $55-71 \%$ of the total patients included in the pivotal trials and $35-41 \%$ in Korean study.

Also, another even more remarkable difference was that the IFNL4 gene (previous mistakenly refer to IL28B, rs12979860) C/C constituted the $33-47 \%$ of total study population in the pivotal study patients. ${ }^{4,5}$ It is a strong predictor of response to PEG-IFNbased therapy. ${ }^{16}$ Individuals with the favorable $\mathrm{C} / \mathrm{C}$ allele have about a 2-fold higher likelihood of achieving SVR compared to individuals with $C / T$ or $T / T$ genotype. For specific treatment regimen including sofosbuvir, the IFNL4 variant still influences treatment outcomes although the SVR remains relatively high for all IFNL4 genotypes. For example, a trial for the treatment-naïve genotype $1,4,5,6$ patients treated with 12 weeks of SOF+PEG-IFN+R (NEUTRINO study), ${ }_{1}^{4}$ the SVR rates were $98 \%$ in individuals with $\mathrm{C} / \mathrm{C}$ allele and $87 \%$ in individuals with non- $\mathrm{C} / \mathrm{C}$ allele. The individuals in this study had genotype 1 or 4 HCV infection and received SOF+PEG-IFN+R. The frequency of the rs12979860 favor- 
able C/C alleles varies broadly across different populations, allele frequency was nearly 0.9 in East Asians, followed by Caucasians (0.63) and Hispanics (0.55), and is the least common in patients of African origin (0.39). In Korea, this C/C polymorphism was found in $81-88 \%$ of the patients. ${ }^{11,17}$

In genotype 2, clinical significance of IFNL4 gene polymorphism is still uncertain. In FISSION study including genotype 2, 3 treated with SOF $+R_{r}{ }^{4}$ the SVR rate was $69 \%$ in individuals with $C / C$ allele and $66 \%$ in individuals with non-C/C allele. In POSITRON study including IFN-ineligible genotype 2 patients treated with $\mathrm{SOF}+\mathrm{R}{ }^{6}$ the SVR rate was $76 \%$ in individuals with C/C allele and $79 \%$ in individuals with non-C/C allele. In a Japanese study, ${ }_{1}^{13}$ SVR rate were $98 \%$ in subjects with C/C allele and $94 \%$ in non-CC alleles. In a Korean study, ${ }^{11}$ SVR rate were $97 \%$ in subjects with C/C allele and $96 \%$ in non-CC alleles.

Since there are too many DAA treatment options and those SVR rates are excellent, there will be low possibility to prove clinical significance of SOF+R treatment in future trials. Nevertheless, because of the aforementioned reasons including acceptable safety profiles, excellent SVR rate, and possibility of economic advantage, the SOF+R may be the one of the best first line options for genotype 2 chronic hepatitis $C$ patients who cannot receive other DAAs in Korea.

\section{Conflicts of Interest}

The author has no conflicts to disclose.

\section{REFERENCES}

1. Korean Association for the Study of the Liver (KASL). $2017 \mathrm{KASL}$ clinical practice guidelines management of hepatitis C: treatment of chronic hepatitis C. Clin Mol Hepatol 2018;24:169-229.

2. AASLD/IDSA HCV Guidance Panel. Hepatitis C guidance: AASLDIDSA recommendations for testing, managing, and treating adults infected with hepatitis C virus. Hepatology 2015;62:932-954.

3. European Association for the Study of the Liver. EASL recommendations on treatment of hepatitis C 2018. J Hepatol 2018;69:461-511.

4. Lawitz E, Gane EJ. Sofosbuvir for previously untreated chronic hepatitis C infection. N Engl J Med 2013;369:678-679.

5. Zeuzem S, Dusheiko GM, Salupere R, Mangia A, Flisiak R, Hyland $\mathrm{RH}$, et al. Sofosbuvir and ribavirin in HCV genotypes 2 and 3. N Engl J Med 2014:370:1993-2001.

6. Jacobson IM, Gordon SC, Kowdley KV, Yoshida EM, Rodriguez-Torres M,
Sulkowski MS, et al. Sofosbuvir for hepatitis C genotype 2 or 3 in patients without treatment options. N Engl J Med 2013;368:1867-1877.

7. Ippolito AM, Milella M, Messina V, Conti F, Cozzolongo R, Morisco $F$, et al. HCV clearance after direct-acting antivirals in patients with cirrhosis by stages of liver impairment: the ITAL-C network study. Dig Liver Dis 2017;49:1022-1028.

8. Welzel TM, Nelson DR, Morelli G, Di Bisceglie A, Reddy RK, Kuo A, et al. Effectiveness and safety of sofosbuvir plus ribavirin for the treatment of HCV genotype 2 infection: results of the real-world, clinical practice HCV-TARGET study. Gut 2017;66:1844-1852.

9. Mangia A, Susser S, Piazzolla V, Agostinacchio E, De Stefano G, Palmieri $\mathrm{V}$, et al. Sofosbuvir and ribavirin for genotype 2 HCV infected patients with cirrhosis: a real life experience. J Hepatol 2017;66:711-717.

10. Foster GR, Pianko S, Brown A, Forton D, Nahass RG, George J, et al. Efficacy of sofosbuvir plus ribavirin with or without peginterferonalfa in patients with hepatitis C virus genotype 3 infection and treatment-experienced patients with cirrhosis and hepatitis $C$ virus genotype 2 infection. Gastroenterology 2015;149:1462-1470.

11. Ahn SH, Lim YS, Lee KS, Paik SW, Lee YJ, Jeong SH, et al. A phase $3 \mathrm{~b}$ study of sofosbuvir plus ribavirin in treatment-naive and treatment-experienced Korean patients chronically infected with genotype 2 hepatitis C virus. J Viral Hepat 2016;23:358-365.

12. Kao JH, Chien RN, Chang TT, Peng CY, Hu TH, Lo GH, et al. A phase $3 \mathrm{~b}$ study of sofosbuvir plus ribavirin in Taiwanese patients with chronic genotype 2 hepatitis C virus infection. Liver Int 2016;36:1101-1107.

13. Omata M, Nishiguchi S, Ueno Y, Mochizuki H, Izumi N, Ikeda F, et al. Sofosbuvir plus ribavirin in Japanese patients with chronic genotype 2 HCV infection: an open-label, phase 3 trial. J Viral Hepat 2014;21:762-768

14. Lee SW, Lee HL, Han NI, Kim HY, Kim CW, You CR, et al. Real-life experience of sofosbuvir and ribavirin for genotype $2 \mathrm{HCV}$ infected Korean patients: a multicenter cohort study [Abstract]. Clin Mol Hepatol 2017;23(Suppl 2):62-63.

15. Kim YM, Kim SB, Song IH, Lee SH, Kim HS, Lee TH, et al. Efficacy and safety of sofosbuvir plus ribavirin for Korean patients with hepatitis $C$ virus genotype 2 infection: a retrospective multi-institutional study. Clin Mol Hepatol 2018;24:311-318.

16. Dean L. Sofosbuvir therapy and IFNL4 genotype. In: Pratt V, McLeod H, Rubinstein W, Dean L, Kattman B, Malheiro A, eds. Medical Genetics Summaries. Bethesda: National Center for Biotechnology Information (US), 2012. E-book website, <https://www.ncbi.nlm. nih.gov/books/NBK61999>. Accessed Aug 2018.

17. Jung YK, Kim JH, Ahn SM, Yang JW, Park SJ, Kim JW, et al. Role of interleukin 28B-related gene polymorphisms in chronic hepatitis $C$ and the response to antiviral therapy in Koreans. J Clin Gastroenterol 2013;47:644-650. 